\title{
Effects of In-Plane Magnetic Fields on the Electronic Cyclotron Effective Mass and Landé Factor in GaAs-(Ga,Al)As Quantum Wells
}

\author{
E. Reyes-Gómez ${ }^{1}$, C. A. Perdomo Leiva ${ }^{2}$, L. E. Oliveira ${ }^{3}$, and M. de Dios-Leyva ${ }^{1}$ \\ ${ }^{1}$ Dept. of Theor. Physics, Univ. of Havana, San Lázaro y L, Vedado 10400, Havana, Cuba \\ ${ }^{2}$ Departamento de Física, ISPJAE, Calle 127 s/n, Marianao 19390, Havana, Cuba \\ ${ }^{3}$ Instituto de Física, Unicamp, CP 6165, Campinas, São Paulo, 13083-970, Brazil
}

Received on 8 December, 2005

\begin{abstract}
The dependence of the electron Landé $g$-factor on carrier confinement in quantum wells recently gained both experimental and theoretical interest. The $g$ factor of electrons in $\mathrm{GaAs}-(\mathrm{Ga}, \mathrm{Al}) \mathrm{As}$ quantum wells is of special interest, as it changes its sign at a certain value of the well width. In the present work, the effects of an in-plane magnetic field on the cyclotron effective mass and on the Landé $g_{\perp}$-factor in single GaAs-(Ga,Al)As quantum wells are studied. Theoretical calculations are performed in the framework of the effective-mass and nonparabolic-band approximations. The Ogg-McCombe Hamiltonian is used for the conduction-band electrons in the semiconductor heterostructure, and the Landé $g_{\perp}$-factor theoretically evaluated is found in good agrement with available experimental measurements.
\end{abstract}

Keywords: Magnetic fields; Quantum wells; g-factor; Cyclotron effective mass

Semiconductor heterostructures, such as quantum wells (QWs), quantum-well wires (QWWs), quantum dots (QDs), and superlattices (SLs) have been widely studied in the past three decades. Such interest was motivated by possible electronic and opto-electronic applications and by the need to understand fundamental properties of matter at nanoscale dimensions. In that respect, the transport of spin-polarized electrons by using ferromagnetic probe tips in a low-temperature scanning tunnelling microscope opened up the possibility of investigating magnetic systems at spatial resolutions in the angstrom scale [1]. Of course, the ability to manipulate single spins [2] is one of the important aspects in the development of quantum information processing and spintronics. In particular, the dependence of the electronic effective mass and electron Landé $g$-factor on carrier quantum confinement in QWs and QDs has recently gained the community attention both experimentally as well as theoretically [3-8]. The $g$-factor of electrons in GaAs- $\mathrm{Ga}_{1-x} \mathrm{Al}_{x}$ As QWs is of special interest, as it changes its sign at certain values of the well width. In this study we are particularly interested in the experimental work by Hannak et al [4] who determined the electron Landé factor as a function of the GaAs- $\mathrm{Ga}_{1-x} \mathrm{Al}_{x} \mathrm{As}$ well width from 1 to $20 \mathrm{~nm}$ under in-plane magnetic fields by the technique of spin quantum beats. We are also concerned with the experimental data by Le Jeune et al [5] who studied the anisotropy of the electron Lande factor in GaAs QWs, and by Malinowski and Harley [6] who investigated the influence of quantum confinement and built-in strain on conduction-electron $g$ factors in $\mathrm{GaAs} / \mathrm{Al}_{0.35} \mathrm{Ga}_{0.65} \mathrm{As} \mathrm{QWs}$ and strained-layer $\operatorname{In}_{0.11} \mathrm{Ga}_{0.89} \mathrm{As} / \mathrm{GaAs} \mathrm{QWs}$, for QW widths between 3 and $20 \mathrm{~nm}$. Here, we are concerned with the effects of in-plane magnetic fields on the cyclotron effective mass and Landé $g_{\perp}$-factor in $\mathrm{GaAs}-\mathrm{Ga}_{1-x} \mathrm{Al}_{x} \mathrm{As}$ semiconductor QWs, within the effective-mass and non-parabolic-band approximations $[9,10]$, with theoretical results compared with available experimental measurements. Details of the present work will be presented elsewhere [11].

The effective Hamiltonian for the conduction-band electrons in a GaAs- $\mathrm{Ga}_{1-x} \mathrm{Al}_{x} \mathrm{As} \mathrm{QW}$, grown along the $y$ axis, under an in-plane $\mathbf{B}=B \hat{z}$, magnetic field is given by

$$
\begin{aligned}
\hat{H} & =\frac{\hbar^{2}}{2} \hat{\mathbf{K}} \frac{1}{m(y)} \hat{\mathbf{K}}+\frac{1}{2} g(y) \mu_{B} \hat{\sigma}_{z} B+\Gamma \hat{\boldsymbol{\sigma}} \cdot \hat{\tau}+a_{1} \hat{\mathbf{K}}^{4}+\frac{a_{2}}{l_{B}^{4}}+a_{3}\left[\left\{\hat{K}_{x}^{2}, \hat{K}_{y}^{2}\right\}+\left\{\hat{K}_{x}^{2}, \hat{K}_{z}^{2}\right\}+\left\{\hat{K}_{y}^{2}, \hat{K}_{z}^{2}\right\}\right]+ \\
& +a_{4} B \hat{\mathbf{K}}^{2} \hat{\boldsymbol{\sigma}}_{z}+a_{5}\left\{\hat{\boldsymbol{\sigma}} \cdot \hat{\mathbf{K}}, \hat{K}_{z} B\right\}+a_{6} B \hat{\sigma}_{z} \hat{K}_{z}^{2}+V(y),
\end{aligned}
$$

where $\hat{\mathbf{K}}=\hat{\mathbf{k}}+\frac{e}{\hbar c} \hat{\mathbf{A}}, \hat{\mathbf{k}}=-i \nabla ; \hat{\mathbf{A}}=(-y B, 0,0)$ is the magnetic vector potential, $\hat{\sigma}=\left(\hat{\sigma}_{x}, \hat{\sigma}_{y}, \hat{\sigma}_{z}\right)$ are the Pauli matrices, $m(y)$ and $g(y)$ are the growth-direction position-dependent (with bulk values of $\mathrm{GaAs}_{\mathrm{A}}$ or $\mathrm{Ga}_{1-x} \mathrm{Al}_{x} \mathrm{As}$ ) conduction-electron effective mass and Landé $g$-factor, respectively [12], $\mu_{B}$ is the Bohr magneton, $l_{B}$ is the Landau length, $a_{1}, a_{2}, a_{3}, a_{4}, a_{5}$ and $a_{6}$ are constants appropriate to bulk GaAs [13], $\{\hat{a}, \hat{b}\}$ is the anticommutator between the $\hat{a}$ and $\hat{b}$ operators, and $V(y)$ is the confining potential, taken as $60 \%$ of the $\mathrm{Ga}_{1-x} \mathrm{Al}_{x} \mathrm{As}$ and GaAs band-gap offset [14]. The term proportional to $\Gamma$ in (1) is the cubic Dresselhaus spin-orbit term [15].

The eigenfunction of (1) may be chosen as

$$
\left(\begin{array}{l}
\varphi(\mathbf{r}) \\
\phi(\mathbf{r})
\end{array}\right)=\frac{e^{i\left(x k_{x}+z k_{z}\right)}}{\sqrt{S}}\left(\begin{array}{l}
\psi_{n, y_{0}, \uparrow}\left(y-y_{0}\right) \\
\psi_{n, y_{0}, \downarrow}\left(y-y_{0}\right)
\end{array}\right)
$$

where $n$ is the Landau magnetic-subband index, and $y_{0}=k_{x} l_{B}^{2}$ 

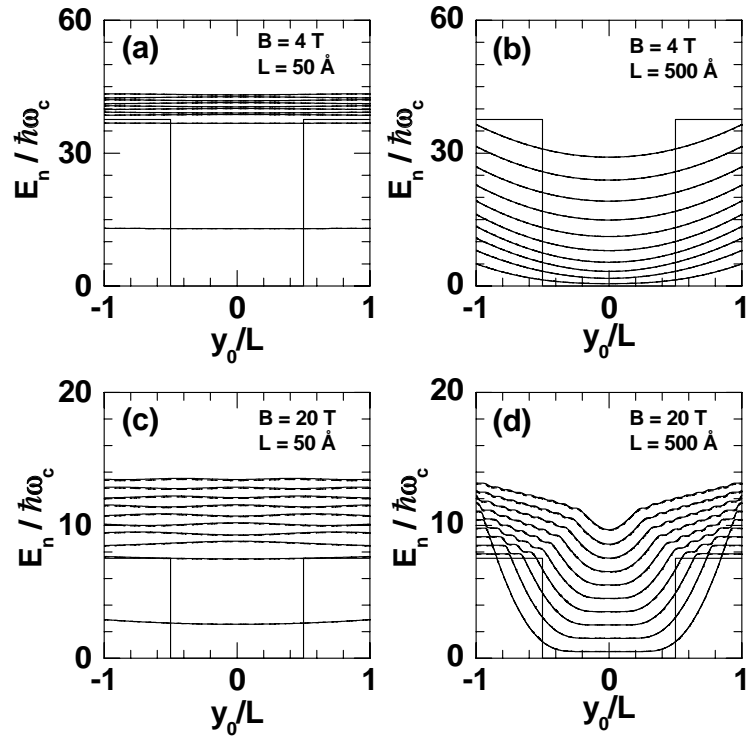

FIG. 1: The ten lowest magnetic levels as functions of the orbitcenter position in GaAs- $\mathrm{Ga}_{0.65} \mathrm{Al}_{0.35} \mathrm{As} \mathrm{QWs}$ of width $L$, under inplane magnetic fields, for $L=50 \AA$ and $B=4 T$ (a), $L=500 \AA$ and $B=4 T$ (b), $L=50 \AA$ and $B=20 T$ (c), and $L=500 \AA$ and $B=20 T(\mathrm{~d})$, respectively. The spin up ( $\uparrow)$ and spin down $(\downarrow)$ states are represented by solid and dashed lines, respectively, although they are essentially undistinguishable for the scale used in the figure. The potential profile is shown schematically.

is the cyclotron orbit-center position. Due to the low population of the conduction states at low temperatures, one may consider only the $k_{z}=0$ contribution to the energy spectra, and by neglecting the off-diagonal terms in (1), the spin up and spin down states become uncoupled. We have denoted as $\hat{H}_{m_{s}}$ the diagonal terms of (1) for a given $m_{s}$ projection ( $\uparrow$ or $\downarrow)$ of the electron spin along the magnetic-field direction, and expanded the corresponding wave functions in terms of the $\left|m, y_{0}\right\rangle$ harmonic-oscillator wave functions [11], i. e.,

$$
\psi_{n, y_{0}, m_{s}}\left(y-y_{0}\right)=\sum_{m} c_{n m}\left(y_{0}, m_{s}\right)\left|m, y_{0}\right\rangle
$$

After some algebraic manipulations, one straightforwardly obtains

$$
\sum_{m}\left(H_{m_{s}}^{(j, m)}-E_{n}\left(y_{0}, m_{s}\right) \delta_{j, m}\right) c_{n, m}\left(y_{0}, m_{s}\right)=0,
$$

and, by diagonalizing $\hat{H}_{m_{s}}$, the eigenvalues $E_{n}\left(y_{0}, m_{s}\right)$. We would like to stress that the terms of order superior to the parabolic $\left(\hat{K}^{2}\right)$ in (1) are quite often taken into account via perturbation theory [16], and in the present work they are exactly considered within the diagonal approach.

Here, the results refer to $\mathrm{GaAs}-\mathrm{Ga}_{0.65} \mathrm{Al}_{0.35} \mathrm{As} \mathrm{QWs}$ under in-plane magnetic fields, as the experimental data from Hannak et al [4], Le Jeune et al [5], and Malinowski et al [6] on the electronic Landé $g_{\perp}$-factor are for $\mathrm{GaAs}-\mathrm{Ga}_{1-x} \mathrm{Al}_{x} \mathrm{As}$ QWs with Al proportion corresponding to $x=0.35$. In Figure 1 we display the ten lowest Landau levels as functions of the orbit-center position in $\mathrm{GaAs}-\mathrm{Ga}_{0.65} \mathrm{Al}_{0.35} \mathrm{As} \mathrm{QWs}$ under an
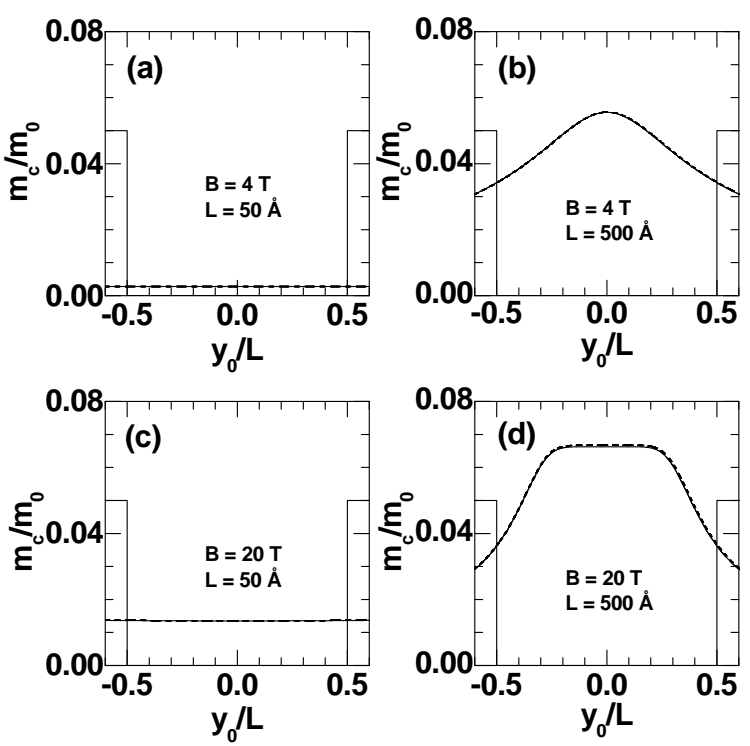

FIG. 2: The cyclotron effective masses as funtions of the orbitcenter position in $\mathrm{GaAs}-\mathrm{Ga}_{0.65} \mathrm{Al}_{0.35} \mathrm{As} \mathrm{QWs}$ under in-plane magnetic fields. Figures (a), (b), (c) and (d) were obtained for $L=50$ $\AA$ and $B=4 T, L=500 \AA$ and $B=4 T, L=50 \AA$ and $B=20 T$, and $L=500 \AA$ and $B=20 T$, respectively. Spin-up and spin-down cases are represented by solid and dashed lines, respectively, although they are undistinguishable in the figure. The potential profile is also shown schematically.
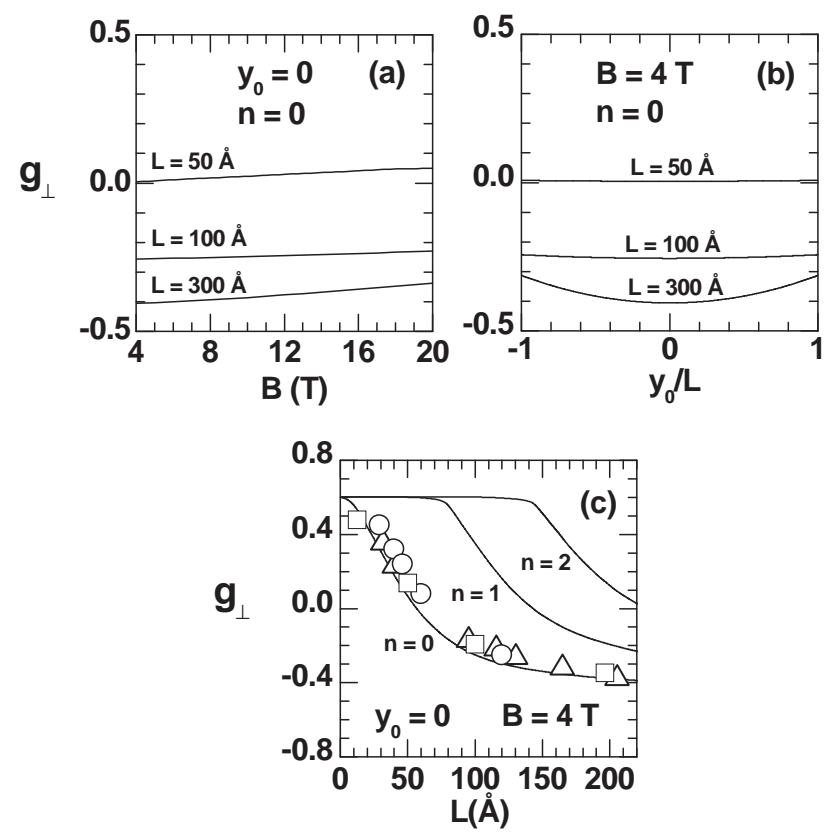

FIG. 3: $g_{\perp}$ - factors, corresponding to the $n^{\text {th }}$ Landau levels, as functions of the applied in-plane magnetic field (a), of the orbit-center position (b), and of the QW width (c) in GaAs- $\mathrm{Ga}_{0.65} \mathrm{Al}_{0.35} \mathrm{As} \mathrm{QWs}$. Squares, circles and triangles in (c) are the experimental results from Hannak et al [4], Le Jeune et al [5], and Malinowski et al [6], respectively. 
in-plane magnetic field of $B=4 T$ and $B=20 T$, and for the QW width of $L=50 \AA$ and $L=500 \AA$. The orbit-center position is in units of the well width $L$, and energies in units of the cyclotron energy $\hbar \omega_{c}=\frac{\hbar e B}{m_{w} c}$, where $m_{w}$ is the conductionelectron effective mass in the well material. Solid and dashed lines are associated with the Landau electron subbands with spin projections in the parallel $(\uparrow)$ and antiparallel $(\downarrow)$ directions of the in-plane applied magnetic field along the $+z$ axis, respectively. Note that the $\uparrow$ and $\downarrow$ electron subbands are essentially undistinguishable in the scale used in Figure 1. Also, as one may notice from Fig. 1 (a), for $B=4 T$ in an $L=50 \AA$ GaAs- $\mathrm{Ga}_{0.65} \mathrm{Al}_{0.35} \mathrm{As} \mathrm{QW}$, and in the range of $y_{0}$ orbit center considered, the lowest Landau energy subbands are essentially flat as a function of the orbit-center position. This behavior is to be expected for small values of the applied magnetic field, as the QW width of $50 \AA$ is small compared with the $l_{B}=128$ $\AA$ Landau length. Therefore, in this case $\left(L<<l_{B}\right)$, the effect of the magnetic field is weak and the electronic Landau energy-level structure is essentially dominated by the barrier confining potential. As the $\mathrm{GaAs}-\mathrm{Ga}_{0.65} \mathrm{Al}_{0.35} \mathrm{As} \mathrm{QW}$ width is increased beyond the $l_{B}$ Landau length, the orbit-center position dependence of the electron Landau subbands becomes dispersive [cf. Fig. 1 (b)], with a minimum at the center of the well, i.e., $y_{0}=0$. At low temperatures, therefore, electrons would tend to populate energy levels around $y_{0}=0$. Notice that, at $B=20 T$, the orbit-center dependence of the electron Landau levels is more dramatic than for $B=4 T$ [cf. Figs. 1 (c) and (d)].

As it is well known, the technique of cyclotron resonance is a powerful tool in the study of the effective mass and transport properties of electrons in semiconductor heterostructures. An in-plane magnetic field may modify the cyclotron effective mass due to the distortion of the Fermi contour by the applied field. In that respect, the inclusion of the band nonparabolicity is crucial in order to obtain a proper quantitative agreement with experimental measurements. For a given projection $m_{s}$ of the electron spin, the $m_{c}$ cyclotron effective mass associated with the $n$-th Landau magnetic subband is defined by $E_{n+1}\left(y_{0}, m_{s}\right)-E_{n}\left(y_{0}, m_{s}\right)=\hbar \frac{e B}{m_{c}}$. The cyclotron effective mass (for $\mathrm{n}=0$ in the above equation) is shown in Figure 2 as a function of the orbit-center position in GaAs- $\mathrm{Ga}_{0.65} \mathrm{Al}_{0.35} \mathrm{As}$ QWs of width $L=50 \AA$, and $L=500 \AA$, under in-plane magnetic fields of $B=4 T$ and $20 T$. In Figs. 2 (a) and (c), the orbit-center position dependence of the cyclotron effective mass is flat, which is due to the fact that the Landau energy levels are essentially independent of the orbit-center position [see Figs. 1 (a) and (c)]. Moreover, the cyclotron effective mass is much smaller [cf. Figs. 2 (a) and (c)] than the bulk GaAs electron effective mass. One may argue that this is due to the large difference between the energies corresponding to the ground and first-excited Landau levels. For $L \gg l_{B}$, the cyclotron effective mass increases and tends to the bulk GaAs electron effective mass for the orbit-center position at the center of the well, as one may see from Figs. 2 (b) and (d). As the magnetic field increases, the effect of the barrier confining potential becomes less important than the magnetic-field confining effect, the cyclotron effective mass increases, and for very large magnetic fields $\left(L \gg l_{B}\right)$, the difference be- tween the $n=1$ and $n=0$ Landau levels is essentially given by $\hbar \omega_{c}=\frac{\hbar e B}{m_{w} c}$, and $m_{c} \rightarrow m_{w}$.

With respect to the $g_{\perp}$-factor, one may define $\Delta E_{n}=$ $E_{n}\left(y_{0}, \uparrow, B\right)-E_{n}\left(y_{0}, \downarrow, B\right)=g_{\perp}^{(n)} \mu_{B} B$, where $g_{\perp}^{(n)}$ is the effective Landé factor in the in-plane direction (perpendicular to the y-growth axis) associated to the $E_{n}\left(y_{0}, m_{s}, B\right)$ Landau level, and the explicit dependence of the Landau levels on the applied in-plane magnetic field is stressed. Notice the above equation is an adequate way of defining the effective $g_{\perp}^{(n)}$-Landé factor associated with the $n$-th Landau magnetic subband and to the two lowest Zeeman $\uparrow$ and $\downarrow$ electronic sublevels. Moreover, it is clear that the effective $g_{\perp}^{(n)}$-factor of the the $n$-th Landau magnetic subband will, in principle, depend on both the orbit-center position and on the applied in-plane magnetic field. Figure 3 shows the $g_{\perp}$ - factor associated to $\uparrow$ and $\downarrow$ spin states in GaAs- $\mathrm{Ga}_{0.65} \mathrm{Al}_{0.35} \mathrm{As}$ QWs. In Fig. 3 (a) we display the magnetic-field dependence of the $g_{\perp}$ - factor corresponding to $n=0$ Landau magnetic levels for various values of the QW width, and for the orbit-center position at the center of the QW. Results were obtained for magnetic fields from $4 T$ to $20 T$. In these range of magnetic-field values, it is apparent that the $g_{\perp}$ - factor depends weakly on the magnetic field. The field-dependence on the $g_{\perp}$ - factor is due both by the modification of the energy band-structure as well as by the redistribution of the wave function by the magnetic field. The orbit-center position dependence of the $g_{\perp}$ - factor associated to the $n=0$ Landau magnetic level is shown in Fig. 3 (b) for $B=4 T$ and for various values of the well width. As the electron-Landau levels are essentially flat for $B=4 T, L=50$ $\AA$ and $L=100 \AA$, for these values of the QW width, the $g_{\perp}$ - factor does not appreciably depend on the orbit-center position. As the QW width increases beyond $l_{B}$, the orbit-center position dependence of the $g_{\perp}$ - factor becomes appreciable. Finally, we show in Fig. 3 (c) the $n=0, n=1$ and $n=2$ Landé $g_{\perp}$ - factors as functions of the QW width for $B=4 T$ and for the orbit-center position at the center of the well (solid lines). For $n=0$, the sign of the electron- $g$ factors in the GaAs well and in the $\mathrm{Ga}_{0.65} \mathrm{Al}_{0.35} \mathrm{As}$ barrier are opposite. For the orbitcenter position at the center of the QW and for short values of the QW-width $\left(L \ll l_{B}\right)$, the electron wavefunctions easily penetrate the $\mathrm{Ga}_{0.65} \mathrm{Al}_{0.35} \mathrm{As}$ barriers, and the $g_{\perp}$ - factors are positive. On the other hand, for large values of the $\mathrm{QW}$ widths $\left(L \gg l_{B}\right)$, the $g_{\perp}$ - factors are negative due essentially to the localization of the electron wavefunctions in the well material. Therefore, there must be a well thickness for which the $g_{\perp}$ - factor is zero, which is clearly observed in Fig. 3 (c). The experimental results from Hannak et al [4], Le Jeune et al [5], and Malinowski et al [6] for the $n=0$ Landau magnetic levels are also represented by squares, circles and triangles, respectively. One may notice that the present theoretical calculations are in excellent agreement with the experimental measurements.

In conclusion, we have studied the effects of an in-plane magnetic field on the cyclotron effective mass and Landé $g_{\perp}$ factor in single GaAs- $(\mathrm{Ga}, \mathrm{Al}) \mathrm{As} \mathrm{QWs}$. Present theoretical results where carried out in the framework of the effectivemass approximation, with the non-parabolic conduction-band 
effects taken into account via the Ogg-McCombe effective Hamiltonian for the conduction electrons. The QW-width dependence $g_{\perp}$ - factor reveals, as expected, a change in its sign, a fact which may be understood in terms of the electron wave function localization. Present theoretical calculations for the Landé $g_{\perp}$-factor in single GaAs-(Ga,Al)As quantum wells were found in excellent agreement with the experimental measurements reported by Hannak et al [4], Le Jeune et al [5], and Malinowski et al [6].

\section{Acknowledgments}

The authors would like to thank the Brazilian Agencies CNPq, FAPESP, Rede Nacional de Materiais Nanoestruturados/CNPq, and MCT - Millenium Institute for Quantum Computing/MCT for partial financial support. MdDL and ERG wish to thank the warm hospitality of the Institute of Physics, State University of Campinas, Brazil, where part of this work was performed.
[1] S. Heinze, M. Bode, A. Kubetzka, O. Pietzsch, X. Nie, S. Blugel, and R. Wiesendanger, Science 288, 1805 (2000); Z. Nussinov, M. F. Crommie, and A. V. Balatsky, Phys. Rev. B 68, 085402 (2003).

[2] I. Zutic, J. Fabian, and S. Das Sarma, Rev. Mod. Phys. 76, 323 (2004); H-A. Engel and D. Loss, Science 309, 586 (2005), and references therein.

[3] R. J. Nicholas, M. A. Hopkins, D. J. Barnes, M. A. Brummell, H. Sigg, D. Heitmann, K. Ensslin, J. J. Harris, C. T. Foxon, and G. Weimann, Phys. Rev. B 39, 10955 (1989); S. Huant, A. Mandray, and B. Etienne, Phys. Rev. B 46, 2613 (1992); B. E. Cole, J. M. Chamberlain, M. Henini, T. Cheng, W. Batty, A. Wittlin, J. A. A. J. Perenboom, A. Ardavan, A. Polisski, and J. Singleton, Phys. Rev. B 55, 2503 (1997).

[4] R. M. Hannak, M. Oestreich, A. P. Heberle, W. W. Ruhle, and K. Kohler, Sol. Stat. Comm. 93, 313 (1995).

[5] P. Le Jeune, D. Robart, X. Marie, T. Amand, M. Brosseau, J. Barrau, V. Kalevich, and D. Rodichev, Sem. Sci. Tech. 12, 380 (1997).

[6] A. Malinowski and R. T. Harley, Phys. Rev B 62, 2051 (2000).

[7] G. Medeiros-Ribeiro, M. V. B. Pinheiro, V. L. Pimentel, and E. Marega, Appl. Phys. Lett. 80, 4229 (2002); R. Hanson, B. Witkamp, L. M. K. Vandersypen, L. H. Willems van Beveren, J. M. Ellerman, and L. P. Kouwenhoven, Phys. Rev. Lett. 91, 196802 (2003); Y. K. Kato, R. C. Myers, A. C. Gossard, and D. D. Awschalom, Science 306, 1910 (2004); A. S. Bracker, E. A. Stinaff, D. Gammon, M. E. Ware, J. G. Tischler, A. Shabaev, Al. L. Efros, D. Park, D. Gershoni, V. L. Korenev, and I. A. Merkulov, Phys. Rev. Lett. 94, 047402 (2005).
[8] E. I. Rashba and A. L. Efros, Phys. Rev. Lett. 91, 126405 (2003); R. de Sousa and S. Das Sarma, Phys. Rev. B 68, 155330 (2003); S. J. Prado, C. Trallero-Giner, A. M. Alcalde, V. LopezRichard, and G. E. Marques, Phys. Rev. B 69, 201310(R) (2004); C. F. Destefani and S. E. Ulloa, Phys. Rev. B 71 161303(R) (2005).

[9] N. R. Ogg, Proc. Phys. Soc. 89, 431 (1966); B. O. McCombe, Phys. Rev. 181, 1206 (1969); M. Braun and U. Rössler, J. Phys. C: Solid State Phys. 18, 3365 (1985).

[10] J. Sabín del Valle, J. López-Gondar, and M. de Dios-Leyva, Phys. Stat. Sol. (b) 151, 127 (1989); A. Bruno-Alfonso, L. Diago-Cisneros, and M. de Dios-Leyva, J. Appl. Phys. 77, 2837 (1995).

[11] M. de Dios-Leyva, E. Reyes-Gómez, C. A. Perdomo-Leiva, and L. E. Oliveira, Phys. Rev. B (in press).

[12] E. H. Li, Physica E 5, 215 (2000); C. Hermann and C. Weisbuch, Phys. Rev. B 15, 823 (1977).

[13] V. G. Golubev, V. I. Ivanov-Omskii, I. G. Minervin, A. V. Osutin, and D. G. Polyakov, Sov. Phys. JETP 61, 1214 (1985).

[14] R. C. Casey, Jr., J. Appl. Phys. 49, 3684 (1978).

[15] G. Dresselhaus, Phys. Rev. 100, 580 (1955).

[16] G. Lommer, F. Malcher, and U. Rossler, Phys. Rev. B 32, 6965 (1985); F. Malcher, G. Lommer, and U. Rossler, Superl. and Microstruct. 2, 267 (1986); G. Lommer, F. Malcher, and U. Rossler, Superl. and Microstruct. 2, 273 (1986); N. Kim, G. C. La Rocca, and S. Rodriguez, Phys. Rev. 40, 3001 (1989); B. Das, S. Datta, and R. Reifenberger, Phys. Rev. 41, 8278 (1990). 